\title{
Intraoperative Hyperoxygenation May Negatively Affect Postoperative Cognitive Functions in Coronary Artery Bypass Graft Operations: A Randomized Controlled Study
}

\author{
TUĞBA ONUR ${ }^{1}$, Ümran Karaca ${ }^{1}$, Filiz Ata ${ }^{2}$, Halil Sayan ${ }^{1}$, Anıl Onur ${ }^{1}$, Canan Yılmaz ${ }^{1}$ \\ Ayşe Balkaya ${ }^{1}$, and Cuneyt Eris ${ }^{2}$ \\ ${ }^{1}$ University of Health Sciences Bursa Yüksek İhtisas Training and Research Hospital \\ ${ }^{2}$ Bursa Yuksek Ihtisas Education and Research Hospital
}

June 7, 2021

\begin{abstract}
Objective: In this study, it was aimed to compare various parameters during surgery and postoperative cognitive functions in patients undergoing coronary artery bypass graft $(\mathrm{CABG})$ surgery using different levels of perioperative oxygen. Methods: One hundred patients scheduled for elective CABG surgery were included in the study. The patients were divided into two groups according to arterial oxygen levels during surgery. In the normoxia group (NG) ( $\mathrm{n}=50), \mathrm{PaO} 2$ was between [?]100mmHg and $<180 \mathrm{mmHg}$ with at least $40 \% \mathrm{FiO} 2$ mechanical ventilation (MV), and in the hyperoxia group $(\mathrm{HG})(\mathrm{n}=50), \mathrm{PaO} 2 \mathrm{was}$ [?]180 mmHg with 100\% FiO2 MV. Hemodynamic parameters, peripheral oxygen saturation (SpO2), cerebral oxygen saturation (rSO2) measured from bilateral sensors, and blood gas values were recorded at the planned measurement times. Mini-mental state examination test (MMSE) was applied to the patients before and at the 12th, 24th hours; on the 1st, 3rd, 6th months after surgery. Results: Extubation time was found to be shorter in NG ( $\mathrm{p}<0.05)$. Between the groups, rSO2 and mean arterial pressure (MAP) were found to be significantly lower in HG at the time of T4 measurement $(\mathrm{p}=0.042, \mathrm{p}=0.038$, respectively). MMSE values of the groups at the 1st, 3rd and 6th months were found to be significantly higher in NG (respectively $\mathrm{p}=$ $0.017, \mathrm{p}=0.014, \mathrm{p}=0.002)$. Conclusion: Hyperoxemia application during CABG may be associated with worse postoperative late-term cognitive functions. Keywords: cardiopulmonary bypass; hyperoxia; postoperative complications; cognitive function; spectroscopy, near-infrared.
\end{abstract}

\section{Hosted file}

2- title page.rtf available at https://authorea.com/users/418372/articles/525143intraoperative-hyperoxygenation-may-negatively-affect-postoperative-cognitive-functionsin-coronary-artery-bypass-graft-operations-a-randomized-controlled-study

\section{Hosted file}

3- abstract.rtf available at https://authorea.com/users/418372/articles/525143intraoperative-hyperoxygenation-may-negatively-affect-postoperative-cognitive-functionsin-coronary-artery-bypass-graft-operations-a-randomized-controlled-study

\section{Hosted file}

4- manuscript.rtf available at https://authorea.com/users/418372/articles/525143intraoperative-hyperoxygenation-may-negatively-affect-postoperative-cognitive-functionsin-coronary-artery-bypass-graft-operations-a-randomized-controlled-study 


\section{Hosted file}

5- references.docx available at https://authorea.com/users/418372/articles/525143intraoperative-hyperoxygenation-may-negatively-affect-postoperative-cognitive-functionsin-coronary-artery-bypass-graft-operations-a-randomized-controlled-study

\section{Hosted file}

TABLE 1.rtf available at https://authorea.com/users/418372/articles/525143-intraoperativehyperoxygenation-may-negatively-affect-postoperative-cognitive-functions-in-coronaryartery-bypass-graft-operations-a-randomized-controlled-study

\section{Hosted file}

TABLE 2.rtf available at https://authorea.com/users/418372/articles/525143-intraoperativehyperoxygenation-may-negatively-affect-postoperative-cognitive-functions-in-coronaryartery-bypass-graft-operations-a-randomized-controlled-study

\section{Hosted file}

TABLE 3.rtf available at https://authorea.com/users/418372/articles/525143-intraoperativehyperoxygenation-may-negatively-affect-postoperative-cognitive-functions-in-coronaryartery-bypass-graft-operations-a-randomized-controlled-study

\section{Hosted file}

TABLE 4.rtf available at https://authorea.com/users/418372/articles/525143-intraoperativehyperoxygenation-may-negatively-affect-postoperative-cognitive-functions-in-coronaryartery-bypass-graft-operations-a-randomized-controlled-study

\section{Hosted file}

TABLE 5.rtf available at https://authorea.com/users/418372/articles/525143-intraoperativehyperoxygenation-may-negatively-affect-postoperative-cognitive-functions-in-coronaryartery-bypass-graft-operations-a-randomized-controlled-study

\section{Hosted file}

figure.docx available at https://authorea.com/users/418372/articles/525143-intraoperativehyperoxygenation-may-negatively-affect-postoperative-cognitive-functions-in-coronaryartery-bypass-graft-operations-a-randomized-controlled-study 